\author{
SERIES "RECENT DEVELOPMENTS IN PULMONARY INFECTIONS" \\ Edited by M. Woodhead and T. Schaberg \\ Number 1 in this series
}

\title{
Chlamydia pneumoniae and the lung
}

\author{
M.R. Hammerschlag
}

\begin{abstract}
Chlamydia pneumoniae and the lung. M.R. Hammerschlag. C ERS Journals Ltd 2000. ABSTRACT: Chlamydia pneumoniae is a frequently occurring respiratory pathogen affecting all age groups. It may cause 5-20\% of community-acquired pneumonias in adults and children. The organism has also been implicated as an infectious trigger for asthma. Furthermore, new studies suggest that it may play a role in the pathogenesis of several chronic diseases including atherosclerosis.

However, despite the growing significance of $C$. pneumoniae as a pathogen, progress is hampered by the lack of standardized diagnostic methods including serology and polymerase chain reaction. This makes it practically impossible for the practitioner to make a specific microbiological diagnosis. The lack of standardized methods has also had an adverse effect on treatment trials. The dependence on serology for diagnosis in treatment studies has generated some questionable results.

Unless cultures are performed, microbiological efficacy cannot be assessed and it may never be possible to survey for or document the emergence of resistance. Eur Respir J 2000; 16: 1001-1007.
\end{abstract}

Division of Infectious Diseases, Dept of Paediatrics, State University of New York Health Science Center at Brooklyn, Brooklyn, NY, USA.

Correspondence: M.R. Hammerschlag, 450 Clarkson Avenue, P.O. Box 49, Brooklyn, New York, 11203-2098, USA. Fax: 71812452118.

Keywords: Asthma, Chlamydia pneumoniae, community-acquired pneumonia, pulmonary infection

Received: January 62000 Accepted: January 62000
Chlamydia pneumoniae was first described as a respiratory tract pathogen by GRAYSTON et al. [1] in 1986. The genus Chlamydia comprises a group of obligate intracellular parasites that have a unique developmental cycle with morphologically distinct infectious and reproductive forms. All members of the genus have a Gram-negative envelope without peptidoglycan, share a genus-specific lipopolysaccharide (LPS) antigen and utilize host adenosine triphosphate for the synthesis of chlamydial protein. The genus now contains four species, $C$. psittaci, $C$. trachomatis, $C$. pneumoniae and $C$. pecorum.

\section{Epidemiology of respiratory infection due to Chlamydia pneumoniae}

C. pneumoniae appears to be a primary human pathogen. Attempts to identify zoonotic reservoirs have been unsuccessful, although $C$. pneumoniae infection has been described in several nonhuman species including koalas [2]. The mode of transmission remains uncertain but is probably via infected respiratory secretions. C. pneumoniae can survive in small aerosol droplets $[3,4]$. There is a report of a laboratory accident in which transmission by aerosol is thought to have taken place [5]. Outbreaks of $C$. pneumoniae have occurred in enclosed populations such as among military recruits and residents of nursing homes [6-8]. Spread of infection has also been documented among family members in the same household [9]. Serological surveys demonstrate a rising prevalence of antibodies directed against $C$. pneumoniae with increasing age, from $10 \%$ at $5-10$ yrs of age, reaching $30-45 \%$ by adolescence and often exceeding $80 \%$ in the elderly [1].
The proportion of community-acquired pneumonias in children and adults associated with $C$. pneumoniae infection has ranged $6-22 \%$, varying with geographical location, age group examined and diagnostic methods used [10]. Most of these studies were based on serology alone.

The term "atypical" pneumonia has been used to differentiate infections caused by C. pneumoniae, Mycoplasma pneumoniae, Legionella and other related organisms from pneumonia caused by classic bacteria, the prototype being Streptococcus pneumoniae. However, recent studies have demonstrated that the clinical presentation of pneumonia due to atypical pathogens cannot readily be differentiated from that caused by "typical" bacteria [10]. This is further complicated by the observation that coinfections with atypical pathogens and other bacteria are frequent. Nonetheless, the term "atypical" can be useful as these organisms share a number of characteristics that separate them from "typical" bacteria. They are either obligate or facultative intracellular parasites that cannot be isolated using routine microbiological methods. The most commonly used method of diagnosis of these infections is serology, which basically offers a retrospective diagnosis. There are no US Food and Drug Administration-approved commercially available nonculture tests for C. pneumoniae and M. pneumoniae.

There is still considerable controversy regarding the significance of atypical pathogens, including C. pneumoniae, as agents of community-acquired pneumonia. Overall, atypical pathogens have been implicated as causing $2-$ $30 \%$ of community-acquired pneumonia in adults and children [10]. The prevalences for individual pathogens can vary greatly from study to study depending upon the

For editorial comments see page 799. 
populations studied and the diagnostic methods used. The results of several selected recent studies are summarized in table 1 . A recently published population-based active surveillance study of community-acquired pneumonia requiring hospitalization in Ohio, USA [11] found that the major three atypical pathogens, $M$. pneumoniae, $C$. pneumoniae and Legionella species, together accounted for $10-38 \%$ depending on the serological criteria used for diagnosis. Individually, the prevalences were $32.5 \%$ for M. pneumoniae, $8.9 \%$ for C. pneumoniae and $3 \%$ for Legionella species compared to 12.6 and $6.6 \%$ for $S$. pneumoniae and Haemophilus influenzae, respectively. Serology was used for the diagnosis of M. pneumoniae and C. pneumoniae infection, and isolation of the organism from sputum or serology for Legionella species. In contrast, diagnosis of bacterial infection required isolation of the organism from blood or pleural fluid (definite infection) or from purulent sputum with supporting Gram-stain appearance (probable infection). A multicentre study in four Scandinavian countries [13] found, using serology exclusively, that $66 \%$ of the patients had a presumptive diagnosis of pneumonia caused by $M$. pneumoniae, chlamydiae or Legionella species. The prevalences for the individual pathogens alone causing infection were 17, 14 and $3 \%$ for M. pneumoniae, C. pneumoniae and Legionella species, respectively. Mixed infections were frequent; an additional $9 \%$ of patients had mixed infection with $M$. pneumoniae and $C$. pneumoniae, and $4 \%$ with $M$. pneumoniae and/or C. pneumoniae and S. pneumoniae. This study differed from the Ohio study in that outpatients as well as inpatients were included; thus the overall severity of infection may have been lower. However, this does not entirely explain the higher prevalence of atypical bacteria and lower prevalence of pneumococcal infection. A smaller US study from Baltimore, MD, USA, conducted during the same time period as the Ohio study, identified infection with an atypical organism in only $7.5 \%$ of the patients, over $50 \%$ of which were also infected with a second pathogen [14]. The diagnostic methods used included culture and polymerase chain reaction (PCR) in addition to serology. Conventional bacterial pathogens were identified in $36 \%$ of the patients.

Although initial serological studies suggested that infection with $C$. pneumoniae was uncommon in young

Table 1. - Role of Chlamydia pneumoniae in communityacquired pneumonia in adults and children: results of selected studies

\begin{tabular}{|c|c|c|c|c|}
\hline $\begin{array}{l}\text { First author } \\
\text { [Ref.] }\end{array}$ & Location & $\underset{n}{\text { Subjects }}$ & $\begin{array}{l}\text { Age } \\
\text { yrs }\end{array}$ & $\begin{array}{l}\text { Prevalence } \\
\text { of } C \text {. } \\
\text { pneumoniae }\end{array}$ \\
\hline MARSTON [11]* & Ohio, USA & 2776 & Adult & 8.9 \\
\hline FILE $[12]^{*}$ & $\begin{array}{c}\text { Multicentre, } \\
\text { USA }\end{array}$ & 456 & Adult & 22 \\
\hline NorRby [13]* & Scandinavia & 383 & Adult & 29 \\
\hline MUNDY $[14]^{+}$ & $\begin{array}{c}\text { Baltimore, MD, } \\
\text { USA }\end{array}$ & 385 & Adult & 3.6 \\
\hline BLOCK $[15]^{+}$ & $\begin{array}{l}\text { Multicentre, } \\
\text { USA }\end{array}$ & 260 & $3-12$ & 13.1 \\
\hline HARRIS [16] & $\begin{array}{c}\text { Multicentre, } \\
\text { USA }\end{array}$ & 456 & $0.5-16$ & 7.3 \\
\hline
\end{tabular}

*: diagnosis based on serology alone; ${ }^{+}$: diagnosis based on culture and/or polymerase chain reaction. children, subsequent studies utilizing culture found the prevalence to be similar to that observed in adults $[15,16]$. As part of a multicentre pneumonia treatment study in children aged 3-12 yrs, BLOCK et al. [15] isolated $C$. pneumoniae from 34 of 260 (13.1\%) children enrolled. Serological evidence of acute infection was found in 48 $(18.5 \%)$, but only eight $(23 \%)$ of the culture-positive children met the serological criteria for acute infection. In a subsequent multicentre study, HARRIS et al. [16] isolated C. pneumoniae from $7.4 \%$ of 420 children, aged $0.5-16$ yrs, with community-acquired pneumonia. Only five $(16 \%)$ of the 31 culture-positive children met the serological criteria for acute infection; most were seronegative. In both studies, the prevalence of culturedocumented $C$. pneumoniae infection was the same in the children who were $<6$ yrs of age as in those who were $>6$ yrs of age. Coinfections with $C$. pneumoniae and other bacteria, especially $S$. pneumoniae, appear to be fairly frequent. Coinfection with $M$. pneumoniae was documented in $20 \%$ of children with culture-documented $C$. pneumoniae infection in the study of BLOCK et al. [15]. Clinically, the children who were infected with both organisms could not be differentiated from those who were infected with either organism alone. The only child in the study of BLOCK et al. [15], who had pneumococcal bactaeremia was also infected with $C$. pneumoniae. These patients frequently respond to treatment with $\beta$-lactam antibiotics, suggesting that $C$. pneumoniae may not be the primary cause of the pneumonia but might disrupt the normal clearance mechanisms, enabling other pathogens to invade. C. pneumoniae has been shown to have a ciliastatic effect on ciliated bronchial epithelial cells in vitro [17].

Persistent nasopharyngeal (NP) infection with $C$. pneumoniae following acute respiratory infection has been documented in adults for periods of up to several years [ 18 , 19]. However, background asymptomatic respiratory infection has been reported in $2-5 \%$ of subjectively healthy adults and children [20-23]. It is not known what role asymptomatic carriage plays in the epidemiology of C. pneumoniae infections.

\section{Clinical presentation}

Most pneumonia due to $C$. pneumoniae appears to be relatively mild and self-limiting and difficult to differentiate clinically from pneumonia due to other organisms such as M. pneumoniae. However exceptions can occur. Acute chest syndrome due to $C$. pneumoniae in children with sickle disease is frequently severe with significant hypoxia [24]. The chest radiograph may show interstitial or lobar infiltrates. Lobar consolidation and pleural effusions also occur. C. pneumoniae has also been isolated from empyema fluid [25] and can cause respiratory failure [26]. Laboratory findings are also nonspecific. The peripheral white blood cell count may be elevated to $\geq 20,000$ cells $\cdot \mathrm{mm}^{-3}$ with a left shift, but is usually $<15,000$ cells $\cdot \mathrm{mm}^{-3}$.

\section{Pathology}

Data regarding histopathological findings in respiratory infection due to C. pneumoniae in humans are not available. However, studies have been performed in animals 
and nonhuman primates. Experimental infection in cynologus monkeys after intranasal inoculation was clinically mild [27]. Chest radiographs 4-6 weeks after inoculation showed no infiltrates. In intranasally inoculated mice, an inflammatory response characterized by infiltrates can be observed in the lungs, with a response dominated predominantly by polymorphonuclear leukocytes in the acute phase and by mononuclear leukocytes in the chronic phase [28]. A characteristic feature is patchy distribution of inflammatory infiltrates interspersed among areas of normal or relatively normal histology. The organism can be seen in the areas of inflammatory reaction. The animals are usually not very ill and the infection resolves spontaneously. After intranasal infection, however, the infection appears to disseminate, probably haematogenously. $C$. pneumoniae can be isolated in culture from not only the lungs but also the spleen as well [28]. The organism has also been cultured from buffy coats of mice after inoculation, and identified in peripheral blood monocytes by PCR [28]. Dissemination following respiratory infection has been hypothesized as the mechanism whereby $C$. pneumoniae may gain access to the vascular system in humans and contribute to the pathogenesis of atherosclerosis. However, haematogenous dissemination of $C$. pneumoniae in the course of respiratory infection has not as yet been demonstrated in humans.

\section{Other pulmonary diseases associated with Chlamydia pneumoniae}

C. pneumoniae has also been implicated in other pulmonary conditions, specifically acute bronchitis, asthma and chronic obstructive pulmonary disease (COPD). A number of studies have been carried out, with contradictory results. Some of this probably relates to how infection with $C$. pneumoniae was defined. Most of the data were based on serology alone.

\section{Acute bronchitis}

Infection with $C$. pneumoniae has been implicated in acute bronchitis [1, 29], although its association with acute exacerbations of chronic bronchitis is less clear. The rate of $C$. pneumoniae infection in patients with acute bronchitis has been reported to range from $2-25 \%$ [30]. As with studies of community-acquired pneumonia, the rate of infection appears to vary depending on the diagnostic methods used. Most studies have been based on serology, using the microimmunofluorescence (MIF) assay. C. pneumoniae has been identified in sputa and throat swab specimens from patients with acute bronchitis by culture and PCR [29]. As seen with $C$. pneumoniae pneumonia, there is no characteristic clinical presentation. Patients are likely to exhibit productive cough, sore throats and hoarseness. In one study, the presence of sore throat and hoarseness, but not cough, was significantly more frequent in patients with $C$. pneumoniae infection [29]. Although it has been suggested that patients with acute bronchitis who test positive for $C$. pneumoniae (usually serologically), the results of several randomized treatment trials with antibiotics active against $C$. pneumoniae (erythromycin and doxycycline) showed no difference in response to therapy between those patients who tested positive and those testing negative [30].

\section{Asthma and chronic obstructive pulmonary disease}

C. pneumoniae may act as an infectious trigger, along with respiratory viruses such as respiratory syncytial virus (RSV), and parainfluenza and M. pneumoniae, for asthma [31]. This organism may be especially well suited for this role because of its ability to cause prolonged infection, often persisting for months. HAMMERSCHLAG et al. [18], reported refractory asthma in a patient with persistent $C$. pneumoniae infection, confirmed by culture, whose symptoms finally resolved after prolonged treatment with erythromycin. The patient was culture-positive for 11 months. Several studies have found association of serological evidence of $C$. pneumoniae infection, and asthma [31, 32]. EmRE et al. [22] isolated C. pneumoniae from the nasopharynx of $11 \%$ of children presenting with an acute episode of wheezing compared to $4.9 \%$ of a control group. Some of the children remained culture-positive for periods of up to 5 months despite treatment, and many were also receiving topical and systemic steroids. The majority of culture-positive children in this study were seronegative by MIF. Similar results in children were reported by CunNingham et al. [33] using PCR and serology. Seventy-five per cent of the children reported on by EMRE et al. [22] who were successfully treated with erythromycin and/or clarithromycin with eradication of the organism showed dramatic clinical improvement in their reactive airway disease. In vitro studies have shown that hydrocortisone enhances the growth of $C$. pneumoniae in vitro, but does not interfere with the antimicrobial activity of macrolides [34]. EMrE et al. [35] subsequently reported that specific anti-C. pneumoniae immunoglobulin (Ig)E could be detected by immunoblotting in $85 \%$ of the sera of children with culture-positive C. pneumoniae infection and wheezing, but was present in only $18-22 \%$ of culture-negative asthmatics or culture-positive children with pneumonia who were not wheezing. The presence or absence of $\operatorname{IgE}$ was not associated with the presence or absence of anti-C. pneumoniae $\operatorname{IgG}$ or IgA as determined by MIF. These findings suggest that type 1 allergy may be implicated in the pathogenesis of reactive airway disease associated with $C$. pneumoniae infection, similar to what appears to occur after viral infection (RSV and parainfluenza).

However, several subsequent studies did not find a significant association of $C$. pneumoniae infection and asthma in adults. CoOK et al. [36] found antibody titres consistent with acute infection in $5.7 \%$ of patients with acute asthma and $5.7 \%$ of controls and serological evidence of past infection in $14.6 \%$ of patients with acute asthma and $12.7 \%$ of controls. LARSEN et al. [37] found that patients with asthma and healthy controls did not differ in prevalence of specific anti-C. pneumoniae IgE or previous $C$. pneumoniae infection as determined by the presence of anti-C. pneumoniae IgG. None of the patients or controls showed serological evidence of acute infection. $C$. pneumoniae neither induced nor enhanced the release of histamine from basophil leukocytes of patients or controls. These results are similar to experience with adults with asthma in Brooklyn, New York, where only $2 \%$ were found to be culture-positive compared to $3 \%$ of controls, and there were no differences in serological evidence of infection, including $\operatorname{IgA}$ and $\operatorname{IgE}$, between cases and controls (S. Weiss, State University of New York, 
USA. Unpublished data). Grayston et al. [29] found that college students with $C$. pneumoniae-associated pneumonia or bronchitis did not wheeze any more frequently than patients with pneumonia or bronchitis who did not have $C$. pneumoniae infection. These data suggest that, if C. pneumoniae plays a role in exacerbations of asthma, it may be more significant in early childhood. Confirmation of an association may require well-controlled prospective studies utilizing culture and/or PCR as well as serology with assessment of the response to specific therapy including demonstration of eradication of the organism.

Serological evidence of $C$. pneumoniae infection has been reported in $7.3->63 \%$ of patients with COPD [38, 39]. Identification of the organism in respiratory samples from these patients by culture or PCR has been infrequent, and most studies have relied on serology alone. BLASI et al. [39], reported that, despite serological evidence of infection being frequent in these patients, only $4 \%$ of exacerbations may be associated with $C$. pneumoniae infection.

\section{Diagnosis}

C. pneumoniae can be isolated from the NP and throat swabs, sputum and pleural fluid of patients with pneumonia, bronchitis and asthma. The nasopharynx appears to be the optimum site for isolation of the organism, especially in children. The relative yield from sputum is not known; however, many patients with $C$. pneumoniae infection may not have productive cough. This is especially true in children. Initial studies suggested that $C$. pneumoniae was very difficult to isolate in tissue culture as compared with C. trachomatis. Originally the same methods were used, HeLa or McCoy cells pretreated with diethylaminoethyl (DEAE) dextran. However, C. pneumoniae grows more readily in other cell lines derived from respiratory tissue, specifically HEp-2 and HL cells [40]. Culture with an initial inoculation and one passage should take 4-7 days.

Although PCR holds promise as a rapid diagnostic test, there are no standardized PCR or other nucleic acid amplification tests for detection of $C$. pneumoniae. The assays currently described in the literature are all in-house tests that employ different primers; the most frequently used have been those based on the omp 1 gene, the $16 \mathrm{~S}$ ribosomal ribonucleic acid (rRNA) gene and a C. pneumoniae-specific deoxyribonucleic acid (DNA) fragment [41]. Some assays are nested, and different methods of detection are used. None have been extensively evaluated compared to culture in respiratory specimens; thus the performance of these assays can vary greatly between studies. A PCR-enzyme immunoassay using 16-S rRNAbased primers was compared to culture for detection of $C$. pneumoniae in NP specimens from 43 symptomatic and 58 asymptomatic individuals [42], PCR had a sensitivity compared to culture of $73 \%$ and a specificity of $99 \%$. In contrast, other investigators using the same primers found that PCR was significantly more sensitive than culture for throat swabs, with 15 of 368 (4\%) specimens from children with respiratory illness positive by PCR but only one $(0.27 \%)$ culture-positive [41]. In contrast, it has been possible to isolate $C$. pneumoniae by culture from NP swabs of $4.9-5 \%$ of asymptomatic children, a 100 -fold greater sensitivity than in the PCR used in the beforementioned study, suggesting that the culture methods used were suboptimal [21, 22]. Explanations for differences in performance between various studies include the presence of DNA polymerase inhibitors in clinical specimens and suboptimal culture methods [41].

Most investigators to date have relied on serological diagnosis, using the MIF test and the compliment fixation (CF) test. The CF test is genus-specific and has been largely used for diagnosis of lymphogranuloma venereum and psittacosis. Originally, GRAYSTON et al. [1] found that fewer than one-third of hospitalized patients with $C$. pneumoniae infection had the $\mathrm{CF}$ antibody. However, in a recent report of a small outbreak of $C$. pneumoniae infection among University of Washington (Seattle, WA, USA) students, all seven patients with pneumonia had CF titres of $\geq 1: 64$ [42]. GRAYSTON et al. [29] have proposed a set of criteria for serological diagnosis using the MIF infection test which are being used by many laboratories and clinicians. For acute infection, the patient should show a four-fold increase in the IgG titre, a single IgM titre of $\geq 1: 16$ or a single IgG titre of $\geq 1: 512$. Past or preexisting infection is defined as an IgG titre of $\geq 1: 16$ and $<1: 512$. On initial infection, the IgM response should appear $\sim 3$ weeks after the onset of illness and the IgG response at 6-8 weeks. On reinfection, the IgM response may be absent and the IgG response occurs earlier, within 1-2 weeks. Because of the relatively long period until the development of a serological response on primary infection, the antibody response may be missed if convalescent sera are obtained too soon, i.e., $<3$ weeks after the onset of illness. The criteria for use of a single serum sample have not been correlated with the results of culture and are based mainly on data from adults. The antibody response in acute infection may take $>3$ months to develop. Acute culture-documented infection can also occur without seroconversion, especially in children; $\geq 70 \%$ of children with culture-documented infection were seronegative even after 3 months' follow-up $[15,16]$. Several studies have found poor correlation between MIF, serology, culture and PCR, even in adult populations [21, 42]. Background rates of seropositivity can be very high in some populations [21]. A study of asymptomatic C. pneumoniae infection among subjectively healthy adults in Brooklyn, New York, found $81 \%$ to have IgG or IgM titres of $\geq 1: 16$ and $17 \%$ showed evidence of "acute infection", an IgG titre of $\geq 1: 512$ and/or an IgM titre of $\geq 1: 16$ [21]. None of these individuals were culture- or PCR-positive. Some IgG may result from a heterotypic response to other chlamydial species as there are cross-reactions between the major outer membrane protein (MOMP) of the three species as well as cross-reactions due to the generic LPS antigen.

The MIF test is also not standardized; there can be a significant subjective component in performing the assay. A recent study [43] attempted to address the problem of interlaboratory variation in the performance of the MIF test by sending a panel of 22 acute and convalescent sera to 14 different laboratories. Some used an in-house MIF test and several used one of two commercially available kits. The overall agreement of all laboratories was $80 \%$, using one laboratory as the "gold standard". The range was $50-100 \%$ depending on the isotype. Agreement for serodiagnostic criteria were $69 \%$ for negative, $68 \%$ for "chronic" and $87 \%$ for a four-fold increase in IgG titre. 
The lack of reactivity in the MIF assay observed in children may be secondary to the lack of reactivity to the MOMP. When sera from culture-positive but MIF-negative children with respiratory infection were examined by immunoblotting, $>89 \%$ had antibodies directed against a number of $C$. pneumoniae proteins but only $24 \%$ reacted with the MOMP [44]. The MOMP does not appear to be immunodominant in the immune response to $C$. pneumoniae infection, although it has been demonstrated to be immunodominant in C. trachomatis infection. Monoclonal antibodies directed against specific epitopes of the MOMP are neutralizing for $C$. trachomatis but not for C. pneumoniae. The MOMP is the major surface-exposed antigen of $C$. trachomatis and may be the major antigen in the MIF assay. Unfortunately, there was no reactivity to any $C$. pneumoniae protein or combination of proteins that readily differentiated infected from uninfected children [44]. When paired sera were examined, the band patterns remained the same for $>70 \%$ of the children. In the remaining children, changes in the immunoblots of acute-phase sera compared with convalescent sera were unique to each patient. When the same sera were immunoblottted against recent clinical isolates of $C$. pneumoniae, there were differences in the intensity of reaction and the band patterns, indicating possible antigenic diversity between isolates. The recent publication [45] of the complete gene sequence of $C$. pneumoniae may facilitate the development of a more specific and sensitive serological assay.

\section{Treatment}

To date there have been few published data describing the response of respiratory infection due to $C$. pneumoniae to antibiotic therapy. C. pneumoniae is susceptible in vitro to macrolides, tetracyclines and fluoroquinolones (table 2) $[46,47]$. Optimum dose and duration of therapy are uncertain. In vitro activity may not predict in vivo efficacy. Anecdotal data suggest that prolonged therapy (i.e., $\geq 2$ weeks) may be desirable since recrudescent symptoms have been described following 2-week courses of erythromycin and even after 30 days' tetracycline or doxycycline $[1,29]$. Practically all pneumonia treatment studies evaluating new macrolides/azalides and quinolones presented or published to date have used serology alone for diagnosis, essentially limiting themselves to a clinical end point. In 1990, LIPSKY et al. [48] described four patients with bronchitis and pneumonia, treated with a 10-day course of ofloxacin, who were retrospectively identified as showing serological evidence of $C$. pneumoniae infection (four-fold rise in $\mathrm{IgG} / \mathrm{IgM}$ titre, single IgM titre of $\geq 1: 16$ or $\mathrm{IgG}$ titre of $\geq 1: 512$ ). All reportedly demonstrated marked clinical improvement. Based on the minimal inhibitory concentrations (MICs) of ofloxacin $\left(1-2 \mathrm{mg} \cdot \mathrm{mL}^{-1}\right)$ against three laboratory strains, the authors concluded that ofloxacin was effective in these patients as the MICs were less than achievable serum levels. In a subsequent prospective pneumonia treatment study, Plouffe et al. [49] found a clinical response rate of $83 \%$ in those patients showing serological evidence of $C$. pneumoniae infection who were treated with ofloxacin compared to $75 \%$ of those who received standard therapy, which was a $\beta$-lactam antibiotic plus erythromycin or a tetracycline. Similarly, FILE et al. [12] reported a clinical
Table 2. - In vitro activity of various antibiotics against Chlamydia pneumoniae

\begin{tabular}{lc}
\hline & $\begin{array}{c}\text { Minimal inhibitory } \\
\text { concentration } \mu \mathrm{g} \cdot \mathrm{mL}^{-1}\end{array}$ \\
\hline Erythromycin & $0.06-0.25$ \\
Doxycycline & $0.06-0.25$ \\
Azithromycin & $0.05-0.25$ \\
Clarithromycin & $0.004-0.03$ \\
Telithromycin (HMR 3647) & $0.031-2$ \\
Ciprofloxacin & 1.0 \\
Ofloxacin & $0.5-2$ \\
Levofloxacin & $0.25-1$ \\
Sparfloxacin & $0.031-0.125$ \\
Trovafloxacin & $0.5-1$ \\
Moxifloxacin & $0.5-1$ \\
Gatifloxacin & $0.125-0.25$ \\
Gemifloxacin & $0.125-0.25$ \\
Sulphamethoxazole & $>500$ \\
\hline
\end{tabular}

(Data from $[46,47]$.)

cure rate of $98 \%$ among patients showing seroligical evidence of $C$. pneumoniae infection who were treated with levofloxacin compared with $93 \%$ of those treated with ceftriaxone and/or cefuroxime axetil, plus erythromycin or doxycycline at the investigator's discretion. In the latter group, the response rate did not differ between those patients who had erythromycin or doxycycline added to their treatment regimen and those who were treated with a cephalosporin alone. There was also no difference in the response rate among those patients who showed "definite" serological evidence of infection, i.e. a four-fold rise in MIF IgG or IgM titre, compared to those who had "probable" infection, i.e. a single IgG titre of $\geq 1: 512$ or IgM titre of $\geq 1: 32$. The success of the cephalosporin regimens raises some questions about the specificity of the serological criteria as these antibiotics show no or poor activity against Chlamydia species in vitro.

Unfortunately, a number of subsequent treatment studies have claimed microbiological eradication, despite the fact that culture was not performed. A recent study by LEOPHONTE et al. [50] comparing two trovafloxacin regimens to amoxycillin clavulanate for the treatment of acute bronchitis found a similar clinical response rate for all three regimens and stated that $C$. pneumoniae and $M$. pneumoniae were successfully eradicated from $80-100 \%$ of the patients at the end of therapy. However, the investigators state in the methods section that "Atypical respiratory pathogens, which were identified by serologic testing (a 4-fold increase in antibody titre) were presumed eradicated if the patient's clinical response was cure or improvement".

There are only four published treatment studies of pneumonia that have utilized $C$. pneumoniae culture and assessed microbiological efficacy. BLOCK et al. [15] found that treatment with erythromycin suspension eradicated C. pneumoniae from the nasopharynx of $86 \%$ of culturepositive children with community-acquired pneumonia and clarithromycin suspension from $79 \%$. All of these children improved clinically despite persistence of the organism. Persistence was not related to the development of antibiotic resistance as all isolates remained susceptible to erythromycin and clarithromycin during and after treatment [51]. Clarithromycin is 10-100-fold more active than erythromycin in vitro and has superior 
pharmacokinetics and tissue penetration, and yet was not more effective than erythromycin in eradicating $C$. pneumoniae from the respiratory tract. Experience with azithromycin has been similar. In an open noncomparative multicentre pneumonia treatment study [52], adolescents and adults of $\geq 12$ yrs of age were given $1.5 \mathrm{~g}$ azithromycin orally over 5 days. $C$. pneumoniae was eradicated from the nasopharynx of seven of the $10(70 \%)$ culture-positive patients with community-acquired pneumonia after treatment. HARRIS et al. [16] reported that $C$. pneumoniae was eradicated after treatment from the nasopharynx of 19 of $23(83 \%)$ evaluable children with community-acquired pneumonia, 0.5-16 yrs of age, who received azithromycin, and in four of four and seven of seven who received amoxycillin clavulanate and erythromycin, respectively $(p=0.9$, Chisquared test).

The MICs and minimal bactericidal concentrations (MBC) of three of nine isolates obtained after treatment from two of seven persistently infected patients in both studies who were treated with azithromycin increased fourfold after treatment, although they were still within the range considered susceptible to the antibiotic. It is not clear whether this was an isolated event or suggestive of possible development of resistance. All patients improved clinically despite persistence of the organism. The results of two pneumonia treatment studies in adults which evaluated levofloxacin and moxifloxacin found eradication rates of $70-80 \%$ [53, 54]. The MICs and minimum bacterial concentrations of isolates of $C$. pneumoniae from the patients who were microbiological failures in respect of both drugs remained the same before and after treatment.

Although resistance of Chlamydia pneumoniae to quinolones has not as yet been reported, Dessus-Babus et al. [55] described two strains of Chlamydia trachomatis which became resistant to sparfloxacin and ofloxacin after four passages in subinhibitory concentrations of the drugs. Resistance appeared to be due to a point mutation in the gyrA quinolone resistance-determining region. Another possible explanation for persistence of Chlamydia pneumoniae in these studies was that the dose and duration of treatment were not optimal. KuTLIN et al. [56], using continuously infected HEp-2 cells, reported that 6 days' treatment with $4 \mu \mathrm{g} \cdot \mathrm{mL}^{-1}$ ofloxacin or $0.05 \mu \mathrm{g} \cdot \mathrm{mL}^{-1}$ azithromycin, which exceeds achievable serum levels, reduced the concentration of Chlamydia pneumoniae from $1 \times 10^{6}$ to $1 \times 10^{3}$ inclusion forming units. $\mathrm{mL}^{-1}$ but failed to completely eradicate the organism.

\section{References}

1. Grayston JT, Campbell LA, Kuo CC, et al. A new respiratory tract pathogen: Chlamydia pneumoniae strain TWAR. J Infect Dis 1990; 161: 618-625.

2. Jackson M, White N, Giffard P, Timms P. Epizootiology of chlamydia infections in two free-range koala populations. Vet Microbiol 1999; 65: 255-264.

3. Theunissen HJJ, Lemmens-Den Toom NA, Burggraaf A, Stolz E, Michel MF. Influence of temperature and relative humidity on the survival of Chlamydia pneumoniae in aerosols. Appl Environ Microbiol 1993; 59: 2589-2593.

4. Falsey AR, Walsh EE. Transmission of Chlamydia pneumoniae. J Infect Dis 1993; 168: 493-496.

5. Hyman CL, Augenbraun MH, Roblin PM, Schachter J, Hammerschlag MR. Asymptomatic respiratory tract infection with Chlamydia pneumoniae TWAR. J Clin Microbiol 1991; 29: 2082-2083.

6. Berdal BP, Scheel O, Ogaard AR, Hoel T, Gutteberg TJ, Anestad G. Spread of subclinical Chlamydia pneumoniae infection in a closed community. Scand J Infect Dis 1992; 24: 431-436.

7. Ekman MR, Grayston JT, Visakorpi R, Kleemola M, Kuo CC, Saikku P. An epidemic of infections due to Chlamydia pneumoniae in military conscripts. Clin Infect Dis 1993; 17: 420-425.

8. Troy CJ, Peeling RW, Ellis AG, et al. Chlamydia pneumoniae as a new source of infectious outbreaks in nursing homes. JAMA 1997; 277: 1214-1218.

9. Yamazaki T, Nakada H, Sakurai N, Kuo CC, Wang SP, Grayston JT. Transmission of Chlamydia pneumoniae in young children in a Japanese family. J Infect Dis 1990; 162: 1390-1392.

10. Hammerschlag MR. Community-acquired pneumonia due to atypical organisms in adults: diagnosis and treatment. Infect Dis Clin Pract 1999; 8: 232-240.

11. Marston BJ, Plouffe JF, File TM Jr, et al. Incidence of community-acquired pneumonia requiring hospitalization. Arch Intern Med 1997; 157: 1709-1718.

12. File TM Jr, Segrett J, Dunbar L, et al. A multicenter, randomized study comparing the efficacy and safety of intravenous and/or oral levofloxacin versus ceftriaxone and/or cefuroxime axetil in treatment of adults with community-acquired pneumonia. Antimicrob Agents Chemother 1997; 41: 1965-1972.

13. Norrby SR. Atypical pneumonia in the Nordic countries: aetiology and clinical results of a trial comparing fleroxacin and doxycycline. $J$ Antimicrob Chemother 1997; 39: 499-508.

14. Mundy LM, Oldach D, Auwaerter PG, et al. and the Hopkins CAP Team. Implications for macrolide treatment in community-acquired pneumonia. Chest 1998; 113: 1201-1206.

15. Block S, Hedrick J, Hammerschlag MR, Cassell GH, Craft JC. Mycoplasma pneumoniae and Chlamydia pneumoniae in pediatric community-acquired pneumonia: comparative efficacy and safety of clarithromycin vs. erythromycin ethylsuccinate. Pediatr Infect Dis J 1995; 14: 471-477.

16. Harris JS, Kolokathis A, Campbell M, Cassell GH, Hammerschlag MR. Safety and efficacy of azithromycin in the treatment of community-acquired pneumonia in children. Pediatr Infect Dis J 1998; 17: 865-871.

17. Shemer-Avni Y, Lieberman D. Chlamydia pneumoniaeInduced ciliostasis in ciliated bronchial epithelial cells. $J$ Infect Dis 1995; 171: 1274-1278.

18. Hammerschlag MR, Chirgwin K, Roblin PM, et al. Persistent infection with Chlamydia pneumoniae following acute respiratory illness. Clin Infect Dis 1992; 14: 178-182.

19. Dean D, Roblin PM, Mandel L, Schachter J, Hammerschlag MR. Molecular evaluation of serial isolates from patients with persistent Chlamydia pneumoniae infections. In: Stephens RS, Byrne GI, Christiansen G, eds. Chlamydia Infections: Proceedings of the Ninth International Symposium on Human Chlamydia Infection. University of California, San Francisco, 1998; 219-223.

20. Gnarpe J, Gnarpe H, Sundelof B. Endemic prevalence of Chlamydia pneumoniae in subjectively healthy persons. Scand J Infect Dis 1991; 23: 387-388.

21. Hyman CL, Roblin PM, Gaydos CA, Quinn TC, Schachter J, Hammerschlag MR. Prevalence of asymptomatic nasopharyngeal carriage of Chlamydia pneumoniae in subjectively healthy adults: assessment by 
polymerase chain reaction-enzyme immunoassay and culture. Clin Infect Dis 1995; 20: 1174-1178.

22. Emre U, Roblin PM, Gelling M, et al. The association of Chlamydia pneumoniae infection and reactive airway disease in children. Arch Pediatr Adolesc Med 1994; 148: 727-732.

23. Block S, Hammerschlag MR, Hedrick J, et al. Chlamydia pneumoniae in acute otitis media. Pediatr Infect Dis $J$ 1997; 16: 858-862.

24. Miller ST, Hammerschlag MR, Chirgwin K, et al. Role of Chlamydia pneumoniae in acute chest syndrome of sickle cell disease. J Pediatr 1991; 118: 30-33.

25. Augenbraun MH, Roblin PM, Mandel LJ, Hammerschlag MR, Schachter J. Chlamydia pneumoniae with pleural effusion: diagnosis by culture. Am J Med 1991; 91: 437.

26. Rumbak MJ, Baselski V, Belenchia J, Griffin JP. Case report: acute postoperative respiratory failure caused by Chlamydia pneumoniae and diagnosed by bronchoalveolar lavage. Am J Med Sci 1993; 305: 390-393.

27. Holland SM, Taylor HR, Gaydos GA, Kappus EW, Quinn TC. Experimental infection with Chlamydia pneumoniae in nonhuman primates. Infect Immun 1990; 58: 593-597.

28. Kuo CC. Pathologic manifestation of chlamydia infection. Am Heart J 1999; 138: S496-S499.

29. Grayston JT, Aldous MB, Easton A, et al. Evidence that Chlamydia pneumoniae causes pneumonia and bronchitis. J Infect Dis 1993; 168: 1231-1235.

30. Bent S, Saint S, Vittinghoff E, Grady D. Antibiotics in acute bronchitis: a meta-analysis. Am J Med 1999; 107: $62-67$.

31. Anderson P. Pathogenesis of lower respiratory tract infections due to chlamydia, mycoplasma, legionella and viruses. Thorax 1998; 53: 302-307.

32. Hahn DL. Chlamydia pneumoniae, asthma and COPD: what is the evidence? Ann Allergy Asthma Immun 1999; 83: 271-292.

33. Cunningham AF, Johnston SL, Julious SA, Lampe FC, Ward ME. Chronic Chlamydia pneumoniae infection and asthma exacerbations in children. Eur Respir J 1998; 11: 345-349.

34. Tsumura N, Emre U, Roblin P, Hammerschlag MR. Effect of hydrocortisone succinate on growth of Chlamydia pneumoniae in vitro. J Clin Microbiol 1996; 34: 23792381.

35. Emre U, Sokolovskaya N, Roblin PM, Schachter J, Hammerschlag MR. Detection of Anti-Chlamydia pneumoniae $\operatorname{IgE}$ in children with reactive airway disease. $J$ Infect Dis 1995; 172: 265-267.

36. Cook PJ, Davies P, Tunnicliffe W, Ayres JG, Honeybourne D, Wise R. Chlamydia pneumoniae and asthma. Thorax 1998; 53: 254-259.

37. Larsen FO, Norn S, Mordhorst CH, et al. Chlamydia pneumoniae and possible relationship to asthma: serum immunoglobulins and histamine release in patients and controls. APMIS 1998; 106: 928-934.

38. Miyashita N, Niki Y, Nakajima M, Kawane H, Matsushima T. Chlamydia pneumoniae infection in patients with diffuse panbronchiolitis and COPD. Chest 1998; 114: 969-971.

39. Blasi F, Legnani D, Lombardo VM, et al. Chlamydia pneumoniae infection in acute exacerbations of COPD. Eur Respir J 1993; 6: 19-22.

40. Roblin PM, Dumornay W, Hammerschlag MR. Use of Hep-2 cells for improved isolation and passage of Chlamydia pneumoniae. J Clin Microbiol 1992; 30: 1968-1971.

41. Boman J, Gaydos CA, Quinn TC. Molecular diagnosis of
Chlamydia pneumoniae infection. J Clin Micro 1999; 37 : 3791-3799.

42. Gaydos CA, Roblin PM, Hammerschlag MR, et al. Diagnostic utility of PCR-enzyme immunoassay, culture, and serology for detection of Chlamydia pneumoniae in symptomatic and asymptomatic patients. JClin Microbiol 1994; 32: 903-905.

43. Peeling RW, Wang SP, Grayston JT, et al. Chlamydia serology: inter-laboratory variation in micro-immunofluorescence results. Proceedings of the Ninth International Symposium on Human Chlamydia Infection. University of California, San Francisco.

44. Kutlin A, Roblin PM, Hammerschlag MR. Antibody response to Chlamydia pneumoniae infection in children with respiratory illness. $J$ Infect Dis 1998; 177: 720-724.

45. Read TB, Brunham RL, Shen C, et al. Genome sequences of Chlamydia Trachomatis MoPn and Chlamydia Pneumoniae AR39. Nucl Acids Res 2000; 28: 1397-1406.

46. Hammerschlag MR. Antimicrobial susceptibility and therapy of infections caused by Chlamydia pneumoniae. Antimicrob Agents Chemother 1994; 38: 1873-1878.

47. Hammerschlag MR. Activity of quinolones against Chlamydia pneumoniae. Drugs 1999; 58 (Suppl. 2): 78-81.

48. Lipsky BA, Tack KJ, Kuo CC, Wang SP, Grayston JT. Ofloxacin treatment of Chlamydia pneumoniae (strain TWAR) lower respiratory tract infections. $\mathrm{Am} \mathrm{J} \mathrm{Med}$ 1990; 89: 722-724.

49. Plouffe JE, Herbert MT, File TM Jr, Baird I, Parsons JN, Kahn JB. Ofloxacin versus standard therapy in treatment of community-acquired pneumonia requiring hospitalization. Antimicrob Agents Chemother 1996; 40: 11751179.

50. Leophonte P, Baldwin RJT, Pluck N. Trovafloxacin versus amoxicillin/clavulanic acid in the treatment of acute exacerbations of chronic obstructive bronchitis. Eur J Clin Microbiol Infect Dis 1998; 17: 434-440.

51. Roblin PM, Montalban G, Hammerschlag MR. Susceptibilities to clarithromycin and erythromycin of isolates of Chlamydia pneumoniae from children with pneumonia. Antimicrob Agents Chemother 1994; 38: 1588-1589.

52. Roblin PM, Hammerschlag MR. Microbiologic efficacy of azithromycin and susceptibilities to azithromycin of isolates of Chlamydia pneumoniae from adults and children with community-acquired pneumonia. Antimicrob Agents Chemother 1998; 42: 194-196.

53. Hammerschlag MR, Roblin PM. Microbiologic efficacy of levofloxacin for the treatment of community-acquired pneumonia due to Chlamydia pneumoniae. Antimicrob Agents Chemother 2000; 44: 1409.

54. Hammerschlag MR, Roblin PM. Microbiologic efficacy of moxifloxacin for the treatment of community-acquired pneumonia due to Chlamydia pneumoniae. Int $J$ Antimicrob Agents 2000; 15: 149-152.

55. Dessus-Babus S, Bebear CM, Charron A, Bebear C, Barbeyrac B. Sequencing of gyrase and topoisomerase IV quinolone-resistance-determining regions of Chlamydia pneumoniae and characterization of quinolone-resistant mutants obtained in vitro. Antimicrob Agents Chemother 1998; 42: 2474-2481.

56. Kutlin A, Roblin PM, Hammerschlag MR. In vitro activities of azithromycin and ofloxacin against Chlamydia pneumoniae in a continuous-infection model. Antimicrob Agents Chemother 1999; 43: 22682272. 